\title{
CONGLOMERABILITY AND FINITE PARTITIONS
}

\author{
ALAN ZAME \\ (Communicated by Bert E. Fristedt)
}

\begin{abstract}
This paper gives a simplified proof of a generalization of the theorem of Schervish, Seidenfeld and Kadane on the extent of nonconglomerability of finitely additive probability measures.
\end{abstract}

1. Introduction. If $\mu$ is a countably additive probability measure on the set $\Omega, A$ is a measurable subseet of $\Omega$ and $\left\{P_{n}\right\}$ is a partition of $\Omega$ into sets of positive measure, then since $\mu(A)=\sum_{n} \mu\left(A \cap P_{n}\right)$ it follows that

$$
\sup \frac{\mu\left(A \cap P_{n}\right)}{\mu\left(P_{n}\right)} \geq \mu(A) \text {. }
$$

More generally, if $\mu$ is not countably additive and $\mu=t \mu_{f}+(1-t) \mu_{c}$ is its decomposition into its purely finitely additive and countably additive parts, then

$$
\sup \frac{\mu\left(A \cap P_{n}\right)}{\mu\left(P_{n}\right)} \geq(1-t) \mu_{c}(A)
$$

From this it follows that

$$
\sup \left[\mu(A)-\sup _{n} \frac{\mu\left(A \cap P_{n}\right)}{\mu\left(P_{n}\right)}\right] \leq t,
$$

where the sup is over all measurable $A$ and countable partitions $\left\{P_{n}\right\}$ of $\Omega$ into sets of positive measure. In [2], Schervish, Seidenfeld and Kadan prove that if $\mu(\cdot \mid \cdot)$ is a conditional probability for $\mu$ then the sup in (*) in fact equals $t$, where the sup is now over all partitions $\left\{P_{n}\right\}$ of $\Omega$ into measurable sets. This sup is the extent of nonconglomerability of $\mu$. (Another, more self-contained, treatment of this result is given in [1].) The purpose of this paper is to give a simpler proof of a somewhat stronger result.

2. The results. Suppose $(\mu, \Omega, S)$ is a probability measure. A function, which we shall also denote by $\mu$, on $S \times(S \backslash \varnothing)$ is called a conditional probability (cp) for $\mu$ if

(i) for each $A \in S \backslash \varnothing, \mu(\cdot \mid A)$ is a probability measure concentrated on $A$;

(ii) if $A \subset B \subset C \subset \Omega, B \neq 0$, then $\mu(A \mid C)=\mu(A \mid B) \mu(B \mid C)$; and

(iii) $\mu(A \Omega)=\mu(A)$.

These conditions imply

(iii) $^{\prime}$ If $\mu(B) \neq 0$, then $\mu(A \mid B)=\mu(A) / \mu(B)$.

Received by the editors June 27, 1986 and, in revised form, October 6, 1986.

1980 Mathematics Subject Classification (1985 Revision). Primary 60A05.

Key words and phrases. Probability measures, finite additivity, conglomerability.

(C) 1988 American Mathematical Society $0002-9939 / 88 \$ 1.00+\$ .25$ per page 
We may decompose $\mu=t u_{f}+(1-t) \mu_{c}$, where $\mu_{c}$ is a countably additive probability measure on $\Omega$ and $\mu_{f}$ is a purely finitely additive probability measure. We write $n(\mu)=t$.

The extent of nonconglomerability of $\mu, \gamma(\mu)$, is

$$
\sup _{A} \sup _{\left\{P_{n}\right\}}\left[\mu(A)-\sup _{n}\left(\mu\left(A \mid P_{n}\right)\right)\right]
$$

where the first sup is over all measurable $A$ and the second over all countable partitions of $\Omega$ into measurable sets. $\gamma(\mu)$ apparently depends on the $\mathrm{cp}$. In any event, the remark in the introduction shows that $\gamma(\mu) \leq n(\mu)$. The result of [2] is that $\gamma(\mu)=n(\mu)$. In particular, $\gamma(\mu)$ is independent of the cp.

The proof that $\gamma(\mu)=n(\mu)$ may readily be reduced to the special case that $\Omega$ is countable and $S=$ all subsets of $\Omega$. (For completeness, the details are included in the next section.) In this case, we actually have a more general result. Suppose $\mu$ is defined on $S \times \mathcal{F}$, where $\mathcal{F}=$ the nonempty finite subset of $\Omega$, and $\mu$ satisfies conditions (i), (ii), and (iii)' in the definition of cp. Then $\mu$ will be called a finitely defined conditional probability (fdcp). We define $\gamma_{f}(\mu)$ to be $\gamma(\mu)$ with the additional restriction that the sets $P_{n}$ in the partition of $\Omega$ are finite.

THEOREM. If $\Omega$ is a countable set, $\mu$ a probability measure on the subset of $\Omega$, $\mu(\cdot \mid \cdot)$ a fdcp for $\mu$, then $\gamma_{f}(\mu)=n(\mu)$.

We remark that the only role that (iii) ${ }^{\prime}$ plays is in the inequality $\gamma_{f}(\mu) \leq n(\mu)$, as before. The reverse inequality, which is the heart of the matter, does not depend on (iii)'.

3. The basic lemma and proof of the Theorem. The basic lemma, from which the results follow easily and which will be proven in $\S 4$, is the following.

LEMMA. If $\Omega$ is a countable set, $\mu$ a purely finitely additive probability measure on the subset of $\Omega\left(\right.$ i.e., $\mu($ point $)=0$ ) and $\mu(\cdot \mid \cdot)$ is a fdcp for $\mu$, then $\gamma_{f}(\mu)=1$.

Notice that in this case (iii)' is vacuous. The conditional $\mu(\cdot \mid \cdot)$ really has no relation to the measure $\mu$. For instance, if $\nu$ is any measure on $\Omega$ for which $\nu$ (point) $\neq 0$ we could define

$$
\mu(A \mid B)=\nu(A \cap B) / \nu(B) \text { for } B \in \mathcal{F},
$$

and this would give a fdcp for $\mu$.

We shall first show how the theorem followed from this lemma and how the theorem implies the result of [2] when $\Omega$ is not assumed countable.

As remarked before, $\gamma_{f}(\mu) \leq n(\mu)$, so all the proofs consist of finding partitions that give the opposite inequality, $n(\mu) \leq \gamma(\mu)$ or $n(\mu) \leq \gamma_{f}(\mu)$. We may also assume that $n(\mu)>0$, for the countably additive case is clear.

PROOF OF THE THEOREM. Let $\varepsilon>0$; write $\mu=t \mu_{f}+(1-t) \mu_{c}$. Then the fdcp $\mu(\cdot \mid \cdot)$ gives a fdcp $\mu(\cdot \mid \cdot)$ for $\mu_{f}$, since $\mu_{f}$ (point) $=0$. Thus there is a partition $\left\{P_{\Omega}\right\}$ of $\Omega$ into finite sets and a set $A$ with

$$
\mu_{f}(A)-\mu\left(A \mid P_{n}\right) \geq 1-\varepsilon
$$

for each $n$. Then $\mu(A)-\mu\left(A \mid P_{n}\right) \geq t-\varepsilon$.

Proof of The Theorem of Schervish, Seidenfeld and Kadane. Let $\varepsilon>0$. There is a partition $\left\{P_{n}\right\}$ of $\Omega, P_{n}$ measurable, with $\sum \mu\left(P_{n}\right)<(1-t)+\varepsilon / 2$. 
Let $\bar{\mu}$ be $\mu$ restricted to the $\sigma$-algebra generated by the $P_{n}$; equivalently, $\bar{\mu}$ may be viewed as a measure on $\{1,2,3, \ldots\}$ with $\bar{\mu}(\{n\})=\mu\left(P_{n}\right)$. Let $\bar{\mu}=s \bar{\mu}_{f}+(1-s) \bar{\mu}_{c}$ be the decomposition of $\bar{\mu}$. (Notice that $\bar{\mu}_{f}$ and $\bar{\mu}_{c}$ may not be the restrictions of $\mu_{f}$ and $\mu_{c}$ and $s$ may not equal $t$.) Then $\mu(\cdot \mid \cdot)$ and $s>t-\varepsilon / 2$ restricts to a cp $\bar{\mu}(\cdot \mid \cdot)$ for $\bar{\mu}$. There exists a partition $\left\{\bar{Q}_{n}\right\}$ of $\{1,2,3, \ldots\}$ into finite sets and $\bar{A} \subset\{1,2,3, \ldots\}$ with

$$
\bar{\mu}(\bar{A})-\bar{\mu}\left(\bar{A} \mid \bar{Q}_{n}\right) \geq s-\varepsilon / 2 \geq t-\varepsilon
$$

for every $n$. Let $A=\bigcup P_{n}$ and

$$
Q_{m}=\bigcup_{n \in \bar{Q}_{m}}\left(P_{n}\right)
$$

Then $\mu(A)-\mu\left(A \mid Q_{m}\right) \geq t-\varepsilon$.

4. Proof of the Lemma. The setting is that $\mu$ is a finitely additive measure on the subsets of the countable set $S, \mu$ (point) $=0$, and $\mu(\cdot \mid \cdot)$ is a fdcp for $\mu$. We define $A>B$ to mean that $\mu(A \mid A \cup B) \geq \mu(B \mid A \cup B)$. Condition (ii) in the definition of fdcp implies that $\succ$ is transitive and

$$
\text { if } A_{1}, \ldots, A_{n} \text { are pairwise disjoint finite sets, }
$$

$$
B \text { finite, } A_{j} \succ B \text { for each } j \text {, then }
$$

$$
\mu\left(B \mid A_{1} \cup \cdots \cup A_{n} \cup B\right) \leq 1 / n \text {. }
$$

DEFinition. An element or finite subset $A$ of $\Omega$ is infinitely exceedable if $\exists$ pairwise disjoint finite sets $E_{1}, E_{2}, E_{3}, \ldots$, such that $E_{j} \succ A$ for each $j$.

Let $I=\{x \in \Omega: x$ is infinitely exceedable $\}$ and let $\mathcal{N}=\Omega \backslash I$. We note that $A \subset \Omega$ is infinitely exceedable if and only if $A \subset I$.

Let $\varepsilon>0$ and $N$ be a positive integer $>1 / \varepsilon$. We will find a set $A$ with $\mu(A)>$ $1-\varepsilon$ and a partition $P=\left\{P_{j}\right\}$ of $\Omega$, each $P_{j}$ finite, with $\mu\left(A \mid P_{j}\right) \leq 1 / N$.

The construction is split into two cases, depending on whether $\mathcal{N}$ or infinite.

CASE 1. Suppose $\mathcal{N}$ is finite. If $F$ is a finite subset of $I$ then $\exists G_{1}, \ldots, G_{N} \subset I$, each $G_{j}$ finite, with $G_{j} \succ F$. Thus, if $G=G_{1} \cup \cdots \cup G_{N}$, we see that

$$
\text { for every finite subset } F \subset I, \exists \text { finite subset } G \subset I
$$$$
\text { with } \mu(F \mid F \cup G) \leq 1 / N \text {. }
$$

Thus, starting with an arbitrary nonempty finite $F_{1} \subset I$ we may find a sequence of pairwise disjoint finite sets $F_{1}, F_{2}, F_{3}, \ldots$ such that

$$
\begin{aligned}
& \mu\left(F_{1} \cup \cdots \cup F_{n} \mid F_{1} \cup \cdots \cup F_{n+1}\right) \leq 1 / N \\
& \text { for each } N, \text { and } \bigcup F_{j}=I .
\end{aligned}
$$

For $j=1, \ldots, N$ let

$$
A_{j}=\bigcup_{k \equiv j(\bmod n)} F_{k} .
$$

Since $\bigcup_{1}^{N} A_{j}=I$, for some $j_{0}, \mu\left(A_{j_{0}}\right) \leq 1 / N$. Let

$$
\begin{gathered}
P_{1}=F_{j_{0+1}} \cup \cdots \cup F_{j_{0}+N}, \\
P_{2}=F_{j_{0}+N+1} \cup \cdots \cup F_{j_{0}+2 N}, \\
\vdots \\
P_{r}=F_{j_{0}+(r-1) N+1} \cup \cdots \cup F_{j_{0}+r N} \quad \text { for } r \geq 1,
\end{gathered}
$$


and let

$$
P_{0}=\mathcal{N} \cup\left(F_{1} \cup \cdots \cup F_{j_{0}}\right) .
$$

Then $\left\{P_{j}: j \geq 0\right\}$ is a partition of $\Omega$ into finite sets. Let

$$
A=\Omega \backslash\left(A_{j_{0}} \cup F_{1} \cup \cdots \cup F_{j_{0}}\right) .
$$

Then

$$
\mu(A)=1-\mu\left(A_{j_{0}}\right)>1-\varepsilon .
$$

Further,

$\mu\left(A \mid P_{r}\right)=\mu\left(F_{j_{0}+(r-1) N+1} \cup \cdots \cup F_{j_{0}+r N-1} \mid F_{\left.j_{0}+(r-1) N+1\right)} \cup \cdots \cup F_{j_{0}+r N}\right) \leq 1 / N$ by $(* * *)$.

CASE 2. Suppose $\mathcal{N}$ is infinite. Then, if $F \subset \Omega$ is finite $\exists G \subset \Omega, G$ finite, $G \cap F=\varnothing$, such that if $H \subset \Omega \backslash(F \cup G)$ is finite, then $G \succ H$. From this it follows that in fact we can find $G$ with $G \cap F=\varnothing$ such that $H \subset \Omega \backslash(F \cup G)$ finite implies that $\mu(H \mid G \cup H) \leq 1 / N$. Thus, we may find a sequence of pairwise disjoint finite sets $\left(F_{j}\right), \bigcup F_{j}=\Omega$, such that if $H$ is finite, $H \subset \Omega \backslash \bigcup_{1}^{n} F_{j}$, then

$$
\mu\left(H \mid H \cup F_{n}\right) \leq 1 / N .
$$

As before, for $j=1, \ldots, N$ let

$$
A_{j}=\bigcup_{k \equiv j(\bmod N)} F_{k} .
$$

$\exists j_{0}$ such that $\mu\left(A_{j}\right) \leq 1 / N$. This time, let

$$
P_{r}=F_{j_{0}+(r-1) N} \cup F_{j_{0}+(r-1) N+1} \cup \cdots \cup F_{j_{0}+r N-1}
$$

and let $A=\Omega \backslash\left(A_{j_{0}} \cup F_{1} \cup \cdots \cup F_{j_{0}}-1\right)$ (or $\Omega \backslash A_{1}$ if $\left.j_{0}=1\right)$. Then $\mu(A)=1-\mu\left(A_{j_{0}}\right)>$ $1-\varepsilon$. Furthermore, $A \cap P_{r}$ is a finite set contained in $\Omega \backslash\left(F_{1} \cup \cdots \cup F_{j_{0}+(r-1) N}\right)$, so, by $(* * * *)$,

$$
\mu\left(A \mid P_{r}\right) \leq 1 / N \text {. }
$$

REMARK. Although we refer to $n(\mu)$ in terms of the decomposition of $\mu$ as $t \mu_{f}+(1-t) \mu_{c}$, we could, perhaps more simply, define it as $\sup \left(1-\sum \mu\left(P_{n}\right)\right)$, where the sup is taken over all countable measurable partitions of $\Omega$. The sup may or may not be attained by any partition. If it is, then the theorem implies that the sets in the various partitions of the theorem may be finite unions of the sets in some fixed partition, i.e., from the point of view of $\gamma$ the space behaves as a countable set.

\section{BIBLIOGRAPHY}

1. B. M. Hill and D. Lane, Conglomerability and countable additivity, Technical Report \#118, 1983 .

2. M. J. Schervish, T. Seidenfeld and J. Kadane, The extent of non-conglomerability of finitely additive probabilities, Z. Wahrsch. Verw. Gebiete 66 (1984), 205-226.

Department of Mathematics and Computer SCience, University of Miami, CORAL GABLES, FLORIDA 33124 